\title{
REVISÃO/REVIEW
}

\section{CUIDADOS PALIATIVOS EM CONDIÇÕES NEUROLÓGICAS AGUDAS}

\section{PALLIATIVE CARE IN ACUTE NEUROLOGICAL CONDITIONS}

\section{Valéria C. Scavasine 1}

\section{RESUMO}

Pacientes neurológicos terminais apresentam sintomas específicos e requerem cuidados paliativos específicos. Certas condições abruptas, como o Acidente Vascular Encefálico (AVE) e Traumatismo Crânio-Encefálico (TCE), levam a uma ruptura súbita com o estilo de vida anterior do paciente e dificuldade de compreensão da família e da equipe sobre a gravidade do quadro e prognóstico. Mesmo quando a terminalidade da vida não está evidente, diante de uma condição de saúde irreversível, sem possibilidade de resposta a um tratamento específico, a equipe médica deve considerar a introdução de cuidados paliativos, visando a promover conforto, qualidade de vida e dignidade ao paciente e seus familiares.

Descritores: Cuidados paliativos. Acidente vascular cerebral. Traumatismo craniocerebrais.

\section{ABSTRACT}

Terminal neurological patients present specific symptoms and require specific palliative care. Some acute neurological conditions, such as Cerebrovascular Diseases (Stroke) and head trauma, lead to sudden rupture with the patient's previous lifestyle. It may be difficult for the family to understand the severity of the case, and for the assistants to estimate prognosis. Even when terminality is unclear, if there is an irreversible damage and no possibility of response to any specific treatment, the team must consider introducting palliative care, focusing on comfort, quality of life and patient's dignity.

Keywords: Palliative care. Stroke. Craniocerebral trauma. 


\section{INTRODUÇÃO}

No ambiente hospitalar e domiciliar, uma das principais faces da humanização da saúde envolve a introdução de cuidados paliativos. São cuidados totais e ativos, cujo objetivo é proporcionar o alívio de dor e outros sintomas. Não se trata de encurtar nem prolongar o processo de morrer, mas sim, de perceber que a disponibilidade de tratamento não justifica a continuação de cuidados médicos fúteis.

As indicações de cuidados paliativos são inúmeras. Em geral, estão indicados nas enfermidades avançadas, progressivas e incuráveis, com prognóstico de vida inferior a seis meses. Porém, qualquer falta de possibilidade razoável de resposta a um tratamento específico, como ocorre em certas condições neurológicas abruptas, deve redirecionar os esforços da equipe no sentido da busca pela qualidade de vida e pela dignidade. Mesmo que a presença explícita da morte não esteja evidente, deve-se levar em conta o impacto emocional da doença no paciente, na família e na equipe de cuidadores.

Com a evolução da medicina tecnológica, tornase cada vez mais difícil distinguir o paciente que se beneficiará de todo esse suporte avançado, que ainda se encontra numa situação potencialmente curável, daquele que receberá medidas onerosas e sem benefícios, que acabarão por prolongar um processo irreversível. Infelizmente é comum, em certos ambientes intensivos, que sujeito não morra mais na sua hora, mas naquela da equipe de saúde.

Sabemos que muitas doenças neurológicas são incuráveis e implacavelmente reduzem a expectativa de vida. Pacientes neurológicos terminais apresentam diversos sintomas, como dor, depressão, fadiga, câimbras, distúrbios do sono, urgência urinária, constipação, distúrbios de comunicação, crises convulsivas, alterações comportamentais e cognitivas. ${ }^{1}$

Devido a esses sintomas, é lógico que eles necessitam de cuidados paliativos específicos. Dessa forma, modelos tradicionais de cuidados paliativos (como os empregados em oncologia, por exemplo) não suprem as necessidades dos pacientes e famílias que lidam com diagnósticos neurológicos.

\section{ACIDENTE VASCULAR ENCEFÁLICO}

Um estudo realizado na França entre 2008 e 2009 analisou 771.000 pacientes com história de AVE. Destes, 505.000 ficaram com algum grau de seqüela, dos quais $50 \%$ apresentaram dificuldades nas atividades de vida diárias ou não conseguiam andar mais de 500m; $45,3 \%$ tinham dificuldade em pelo menos uma atividade de vida diária; e 11,1\% estavam institucionalizados. ${ }^{2}$

Seja isquêmico ou hemorrágico, o AVE implica numa ruptura abrupta com o estilo de vida anterior. Em suas diversas formas de apresentação, pode causar comprometimento grave da linguagem (como nas afasias), do nível de consciência e até do grau de percepção da própria doença e incapacidade (anosognosia). De tal forma, não raro o grau de seqüela chega a incapacitar o doente para participar das decisões sobre o seu próprio caso. Por outro lado, devido à natureza abrupta da condição, a família pode ter dificuldade em compreender a magnitude e a gravidade do quadro.

Nesse contexto de urgência e instabilidade, fica difícil para a equipe médica prever o grau de seqüela de cada paciente. Antes de optar pela introdução de cuidados paliativos, alguns questionamentos devem ser respondidos pela equipe e divididos com os pacientes e familiares.

\section{O que é um AVE grave?}

Pela Sociedade Francesa de Anestesia e Reanimação2 ${ }^{2}$, todo paciente com AVE admitido em UTI, com comprometimento do nível de consciência, com índice de Barthel estimado $<20$ pode ser esperado que apresente piores desfechos. Além disso, devem ser levados em conta o tamanho, a localização e o mecanismo da lesão cerebral.

\section{O que é um AVE de mau prognóstico?}

Em geral, nos grandes ensaios clínicos, considera-se mau desfecho o paciente que evolui com escala modificada de Rankin (tabela 1) maior do que 3 ou 4. No entanto, numa metanálise sobre craniectomia descompressiva, $E m R=4$ foi considerado aceitável ${ }^{3}$. Klein e colaboradores explicaram o conceito de craniectomia descompressiva a 312 adultos e descobriram que apenas $16 \%$ optariam pela cirurgia, considerando aceitável viver com $\mathrm{EmR}=44$.

Faz-se necessário, porém, dissociar os conceitos de capacidade funcional e qualidade de vida. A qualidade pode ser melhor mesmo nos AVE's mais graves, pois depende de outras variáveis, como estado psicológico, depressão, isolamento social, institucionalização e grau de demência.

Bruna MA e colaboradores ${ }^{5}$ entrevistraram 168 pacientes com Síndrome do Encarceramento. Destes, surpreenderam-se ao descobrir que $72 \%$ estavam felizes, $82 \%$ tinham vida social apreciável e $42 \%$ queriam ser reanimados em caso de parada cardio-respiratória. 0 viés do estudo foi a baixa taxa de pacientes que concordaram em responderam aos questionários, provavelmente aqueles que tinham melhor qualidade de vida.

\section{Como estimar o prognóstico na fase aguda?}

Ventilação mecânica, nutrição artificial e craniectomia descompressiva são estratégias que comprovadamente reduzem mortalidade, mas estão associadas, pela gravidade do quadro de base, à maior incapacidade. 
Tabela 1. Escala Modificada de Rankin

\begin{tabular}{lll}
\hline Escore & Classificação & Descrição \\
\hline 0 & Assintomático & Regressão dos sintomas \\
\hline 2 & Sintomas sem incapacidade & Capaz de realizar suas atividades prévias \\
& Incapacidade leve & $\begin{array}{l}\text { Incapaz de realizar todas suas atividades prévias, } \\
\text { mas capaz de atender suas necessidades }\end{array}$ \\
3 & Incapacidade moderada & $\begin{array}{l}\text { Requer alguma ajuda para suas atividades, mas } \\
\text { deambula sem auxílio }\end{array}$ \\
4 & Incapacidade moderada a & Incapaz de deambular e realizar suas atividades \\
& grave & sem ajuda \\
\hline 6 & Incapacidade grave & $\begin{array}{l}\text { Limitado à cama, incontinência, requer cuidado } \\
\text { de enfermeiros e atenção constante }\end{array}$ \\
\hline
\end{tabular}

Em termos de mortalidade, $37,5 \%$ dos AVE's hemorrágicos evoluem para óbito no primeiro mês, sendo essa proporção significativamente menor no AVE isquêmico (11,3\%). ${ }^{6}$ Nesse último grupo, a situação ameaçadora de vida mais freqüente é a oclusão proximal da Artéria Cerebral Média. Sem descompressão cirúrgica, a “média maligna" apresenta mortalidade de 50-80\%. Outra condição potencialmente fatal é a oclusão de basilar. Sem tratamento trombolítico, a mortalidade ultrapassa $80 \%$. Com trombólise intra-arterial ou endovenosa, a mortalidade cai para $40-50 \%$, sendo que $30 \%$ apresentam bons desfechos (Rankin 0 a 2). 7,8

Ainda na sala de emergência, uma ferramenta útil para estimar a gravidade do quadro é escala NIHSS. Cerca de $75 \%$ dos pacientes com NIHSS>17 evoluem para óbito ou ficam gravemente incapacitados, ao contrário de 1/3 dos pacientes com NIHSS $<17 .{ }^{9}$

No entanto, estimar o prognóstico envolve mais do que estimar o risco de morte e incapacidade. A equipe assistente, paciente e familiares devem obter um entendimento completo sobre todos os aspectos da recuperação, como a habilidade de andar, comunicar-se e tolerância à incapacidade. Para isso, recomenda-se utilizar evidências da literatura ao invés de basear-se em experiências pessoais. Se o médico assistente tiver dificuldade, deve considerar pedir uma segunda opinião de um colega mais experiente. Se, mesmo assim, houver incerteza quanto ao prognóstico, isso deve ser comunicado a família.

\section{Como estruturar o serviço para melhor atender esses pacientes?}

A admissão em Unidade de AVC comprovadamente cursa com melhores desfechos em termos de mortalidade, dependência e necessidade de institucionalização.

Em primeiro momento, é importante reconhecer os pacientes que devem ir para a UTI. Numa situação de emergência, a incerteza do diagnóstico e do prognóstico justifica a pronta entubação e reanimação. Em contrapartida, no paciente já hospitalizado, é necessário sempre discutir com os familiares sobre o grau de investimento, de modo que a decisão de reanimar seja antecipada e discutida em equipe.

Em segundo momento, a equipe de cuidados paliativos deve estar integrada à Unidade de AVC. 10 Isso propiciará melhor atendimento ao paciente, suporte psicológico à família, planejamento do cuidado e controle dos sintomas. Enquanto a equipe neurológica assegura a prática clínica baseada em evidências, a equipe de cuidados paliativos é responsável por engajar a família nas decisões, garantir o timing correto das intervenções e trabalhar com a complexidade e o reconhecimento da terminalidade. Também compete a ela abordar questões como grau de investimento, reanimação e doação de órgãos.

Por fim, a Unidade de AVC tem como função educar pacientes e familiares sobre as seqüelas neurológicas, além de encaminhar para centros especializados de reabilitação, quando necessário. Pode existir, ainda, necessidade de tratamentos específicos, como aplicação de toxina botulínica para espasticidade, prevenção de deformidades e facilitação da higiene. 0 atendimento multidisciplinar, iniciado em nível hospitalar, pode e deve se estender além do internamento, transcendendo em visitas domiciliares focadas no paciente e no cuidador. A continuidade do cuidado após a alta abrange desde questões de infra-estrutura, como adaptação do ambiente, até vigilância de aspectos emocionais do cuidador. 


\section{Como otimizar o conforto desses pacientes?}

Num hospital universitário da Suíça, 42 pacientes com AVE referidos para a Unidade de Cuidados Paliativos foram acompanhados entre 2000 e 2005.11 O NIHSS médio era de 21, refletindo a gravidade dos casos. Os sintomas mais observados no fim da vida foram dispnéia (81\%), dor (69\%) e distúrbios de comunicação (93\%). As causas do óbito foram dividas entre causas neurológicas (38\%), que incluíam hidrocefalia, herniação e recorrência do AVE; complicações específicas do AVE (26\%), como pneumonia broncoaspirativa e tromboembolismo pulmonar; e complicações variadas (36\%), como outras infecções e insuficiência cardíaca. ${ }^{11}$

Dispneia e desconforto respiratório são altamente prevalentes nos pacientes neurológicos terminais. Segundo Rogers e colaboradores, todos os pacientes foram a óbito numa Unidade de AVC tiveram dificuldades respiratórias nas últimas 48 horas de vida. As causas incluem pneumonia broncoaspirativa e insuficiência cardíaca, sendo que insuficiência respiratória contribuiu diretamente para a mortalidade em $78 \%$ dos casos.

Os altos índices de disfagia em lesados neurológicos graves justifica o acúmulo de secreção em vias áreas superiores, com predisposição a infecção secundária. Um achado semiológico freqüente em pacientes moribundos com AVE é a presença de roncos disseminados na ausculta pulmonar, decorrente do acúmulo de secreção nos brônquios, conhecido como "ruído da morte". O manejo das secreções pode ser feito com anti-muscarínicos e opioides, que são efetivos em até $48 \%$ dos pacientes.

Dor acomete 42 a $72 \%$ dos pacientes. Devido a distúrbios de comunicação (afasia, rebaixamento), pode ser difícil caracterizá-la como neuropática ou nociceptiva. As formas mais comuns de dor neuropática são cefaléia, dor central pós-AVE, Síndrome da Dor Regional Complexa tipo II. Já a dor nociceptiva, em geral, responde bem à morfina, e costuma decorrer de distensão abdominal, artralgia, espasticidade e úlceras de pressão.

A dor pode estar diretamente relacionada ao AVE, como a dor associada à heminegligência e a dor talâmica. No caso da heminegligência, estratégias compensatórias como estimulação calórica e pistas visuo-espaciais podem trazer benefícios. Quanto à dor talâmica, pela característica neuropática, deve ser tratada com antidepressivos tricíclicos, anticonvulsivantes ou até bloqueio simpático.

0 delirium, extremamente prevalente em unidades de terapia intensiva, costuma acometer pacientes com idade mais avançada, NIHSS elevado, com predominância nas lesões de hemisfério dominante. Até $1 / 3$ dos casos pode ser prevenido, evitando-se o uso excessivo de sedativos, desidratação, distúrbios do ciclo sono-vigília além de promover estimulação cognitiva e mobilização precoce. Quando identificada, deve ser tratada a causa de base, como distúrbios metabólicos e infecções. Anti-psicóticos em baixa dose podem ser usados por curtos períodos, sendo os benzodiazepínicos reservados apenas para os casos de abstinência alcoólica. A dexmetomidina também vem sendo cada vez mais utilizada no ambiente intensivo.

A Síndrome do Ombro Hemiplégico afeta cerca de $22 \%$ dos sobreviventes de AVE e pode ser identificada em $55 \%$ dos pacientes com AVE em centros de reabilitação. Diminui qualidade de vida e recuperação funcional, podendo levar a sintomas depressivos, distúrbios do sono e hospitalização mais prolongada. Pode aparecer em 2 semanas, porém, seu pico de incidência ocorre em 2 a 3 meses após o AVE.

Trata-se de uma entidade clínica de origem multifatorial, cujas causas incluem subluxação, contraturas musculares, síndrome da dor complexa regional, lesão do manguito rotador, espasticidade e desequilíbrio da articulação gleno-umeral. Via de regra, a flacidez do membro plégico leva a um estiramento da cápsula umeral e predisposição à subluxação. As alterações de tônus e de padrões sinérgicos pré-estabelecidos podem levar à lesão do manguito rotador e instabilidade da escápula. Quando instalada, a Síndrome do Ombro Hemiplégico é de difícil manejo, e o ideal é iniciar o tratamento precocemente. Entre as ferramentas terapêuticas, figuram os antiespásticos (baclofeno), anti-inflamatórios, bloqueios regionais com anestésico, infiltração com corticoide e estimulação elétrica.

Mais de $50 \%$ dos sobreviventes de AVE relatam fadiga após 1 ano. Tal sintoma é mais comum em lesões de tronco, subcorticais e talâmicas. Suspeita-se que o acometimento da formação reticular, presente no tronco encefálico e substância branca subcortical, seja responsável pela clínica de déficit de atenção. A fadiga pode ser manejada, portanto, com amantadina, metilfenidato e modafinila.

A labilidade emocional ou afeto pseudobulbar pode ser observado em $1 / 5$ dos sobreviventes com AVE nos primeiros 6 meses. 0 uso de inibidores seletivos de recaptação de serotonina reduz a freqüência dos episódios de choro e riso. Além disso, a combinação de Dextrometorfano e Quinidina tem apresentado bons resultados.

\section{Traumatismo Crânio-Encefálico}

Os principais fatores de mau prognóstico após TCE grave são: idade superior a 64 anos, Glasgow na admissão inferior a 9, achados na tomografia e exame neurológico.

Um estudo com 96 pacientes idosos portadores de TCE identificou uma taxa de mortalidade geral de $68 \%$, sendo lesões de tronco encefálico e hematomas epidurais as entidades de maior letalidade $(90$ a $100 \%$, respectivamente). Desses 96 pacientes com mais de 64 anos, 1/3 recebeu tratamento cirúrgico, 1/3 tratamento 
Tabela 2. Manejo dos sintomas mais prevalentes nos pacientes com AVE

\begin{tabular}{ll}
\hline Sintoma & Tratamento \\
\hline Incontinência & Treinamento, toxina botulínica, cateterização intermitente \\
Constipação & Lactulose, leite de magnésio, bisacodil \\
\hline Crises Convulsivas & Tratar se $\geq 2$ crises não provocadas \\
\hline Disfunção Sexual & Atendimento psicológico \\
& Estratégias de comunicação \\
\hline Distúrbios respiratórios do & Inibidor da fosfodiesterase? Evidência insuficiente \\
sono & CPAP, BiPAP \\
\hline Depressão & ISRS \\
\hline Ansiedade & Psicoterapia: resultados pobres ${ }^{12}$ \\
\hline
\end{tabular}

suportivo e 1/3 foi encaminhado para cuidados paliativos. Dos que foram paliados, 90\% evoluíram para óbito. 13

Após reconhecer a gravidade, a limitação de suporte passa a ser discutida diante de lesões cerebrais irreversíveis, responsáveis por grave comprometimento do nível de consciência. Frequentemente, num rebaixamento importante, a presença e a intensidade da dor não podem ser mensuradas, surgindo a necessidade de instituir analgesia e sedação paliativas.

Existem critérios para iniciar analgesia paliativa, baseados em marcadores clínicos de dor e ansiedade:

- Elevação de PA

- Taquicardia

- Taquipneia

- Respostas motoras a estímulos não-nocivos

- EEG evidenciando resposta a estímulos nocivos

Diante do paciente neurológico grave, é importante diferenciar o estado de consciência mínima, no qual o sujeito é capaz de sentir dor e ansiedade, do estado vegetativo persistente. Nesse último, o estímulo supostamente não chegaria ao córtex primário, que está desconectado do centro da percepção dolorosa. Pacientes que parecem calmos, ou que não apresentam sinais neurológicos e hemodinâmicos de dor, provavelmente não requerem sedação.

A sedação paliativa em fase final de vida é uma intervenção bem documentada, com indicações precisas e que pode aliviar significativamente $\mathrm{o}$ sofrimento do paciente em fase terminal, sem a instituição de medidas com a finalidade de abreviar sua vida. Pode ser erroneamente interpretada como uma forma disfarçada de eutanásia - prática em que intencionalmente se realizam medidas com a finalidade de abreviar a vida. Tal interpretação incorreta pode ocorrer especialmente quando a necessidade de sedo-analgesia para manejo dos sintomas não é tão explícita. No estado de consciência mínima, por exemplo, há uma dificuldade em se diferenciar respostas reflexas de percepção da dor, e mesmo quando há somente respostas reflexas, ainda existe uma possibilidade de "dor inconsciente". A fim de se evitar qualquer forma de sofrimento, mesmo quando esse não possa ser documentado, a sedação acaba sendo recomendada toda vez que o paciente é retirado da ventilação mecânica, extubado ou decanulado, assim como se deve discutir a interrupção de nutrição e hidratação.

A sedação paliativa inclui o uso de medicamentos com ação hipnótica, com objetivo de reduzir a consciência de um fator estressante, dor ou desconforto. Se possivel, deve-se preservar a capacidade do indivíduo de interagir com o ambiente, titulando-se as medicações e utilizando o menor nível necessário para aliviar o sofrimento. ${ }^{14}$

Não existe evidência de aumento do conforto com hidratação e nutrição artificial. Sabemos que pacientes moribundos voluntariamente param de comer e beber. Além disso, a hidratação desnecessária dificulta o manejo de secreções e aumenta o volume de perdas para o terceiro espaço. Embora não exista consenso sobre hidratação e nutrição parenteral, em geral, evita-se a hiperhidratação, justamente pela dificuldade de manejo das secreções. Além disso, para aumentar o conforto do paciente, costuma-se retirar a dieta na fase final, principalmente ao se observar sinais de gastroparesia (distensão abdominal, vômitos ou estase). A decisão cabe ao médico assistente, mas é fundamental que a família já tenha sido abordada e esteja de acordo com a conduta. 


\section{DISCUSSÃO}

$\mathrm{Na}$ maioria dos países, não é permitido intencionalmente abreviar a vida, embora tratamentos para aliviar sofrimento sejam permitidos, mesmo que não cursem com ações que prolonguem a vida. É importante legitimar quais práticas são permitidas para tranqüilizar os inúmeros pacientes que temem uma "morte ruim" e para uniformizar a prática entre os médicos. De acordo com o Código de Ética Médica, o médico deve aceitar as escolhas de seus pacientes (Princípio XXI), evitar procedimentos diagnósticos e terapêuticos desnecessários (Princípio XXV) e não abandonar o paciente por este ser portador de moléstia crônica, devendo continuar a assisti-lo, a fim de garantir assistência adequada, voltada para cuidados paliativos exclusivos. (Capítulo V - Art 36).

Eticamente e legalmente, a ortotanásia é admitida como conduta a ser adotada diante de pessoas em processo de morte, quando todas as possibilidades terapêuticas foram esgotadas, não havendo, assim, possibilidade de reversão da condição clínica fatal. 15 Até porque o contrário disso pode levar a distanásia, o que é penalmente punível pelo código penal brasileiro.

\section{CONCLUSÃO}

É dever do médico aliviar o sofrimento dos pacientes, honrar seus valores e desejos e ajuda-los a chegar ao fim da vida com dignidade.

\section{REFERÊNCIAS}

1. Boersma I, Miyasaki J, Kutner J, Kluger B. Palliative care and neurology: time for a paradigm shift. Neurology. 2014 Aug 5;83(6):561-7.

2. Woimant $\mathrm{F}$, Biteye $\mathrm{Y}$, Chaine $\mathrm{P}$, Crozier S. Severe stroke: which medicine for which results? Ann Fr Anesth Reanim. 2014 Feb;33(2):102-9.

3. DECIMAL, DESTINY, and HAMLET investigators. Early decompressive surgery in malignant infarction of the middle cerebral artery: a pooled analysis of three randomised controlled trials. Lancet Neurol. 2007 Mar;6(3):215-22.

4. Klein A, Kuehner C, Schwarz S. Attitudes in the general population towards hemi-craniectomy for middle cerebral artery (MCA) infarction. A population-based survey. Neurocrit Care. 2012 Jun;16(3):456-61

5. Bruno MA et al. A survey on self-assessed well-being in a cohort of chronic locked-in syndrome patients: happy majority, miserable minority. BMJ 2011.

6. Xian Y, Holloway RG, Pan W, Peterson ED. Challenges in assessing hospital-level stroke mortality as a quality measure: comparison of ischemic, intracerebral hemorrhage, and total stroke mortality rates. Stroke. 2012 Jun;43(6):1687-90.
7. Archer $\mathrm{CR}$, Horenstein S. Basilar artery occlusion: clinical and radiological correlation. Stroke. 1977 MayJun;8(3):383-90.

8. Lindsberg PJ, Mattle HP. Therapy of basilar artery occlusion: a systematic analysis comparing intraarterial and intravenous thrombolysis. Stroke. 2006 Mar;37(3):922-8.

9. Adams HP Jr, Davis PH, Leira EC, Chang KC, Bendixen $\mathrm{BH}$, Clarke WR, Woolson RF, Hansen MD. Baseline NIH Stroke Scale score strongly predicts outcome after stroke: A report of the Trial of Org 10172 in Acute Stroke Treatment (TOAST). Neurology. 1999 Jul 13;53(1):126-31.

10. Burton CR, Payne S. Integrating palliative care within acute stroke services: developing a programme theory of patient and family needs, preferences and staff perspectives. BMC Palliative Care 2012, 11:22

11. Mazzocato C, Michel-Nemitz J, Anwar D, Michel P. The last days of dying stroke patients referred to a palliative care consult team in an acute hospital. Eur J Neurol. 2010 Jan;17(1):73-7.

12. Hackett ML, Anderson CS, House A, Xia J. Interventions for treating depression after stroke. Cochrane Database Syst Rev. 2008

13. Mitra B, Cameron PA, Gabbe BJ, Rosenfeld JV, Kavar B. Management and hospital outcome of the severely head injured elderly patient. ANZ J Surg. 2008 Jul;78(7):588-92.

14. Baumann A, Claudot F, Audibert G, Mertes PM, Puybasset $L$. The ethical and legal aspects of palliative sedation in severely brain-injured patients: a French perspective. Philosophy, Ethics, and Humanities in Medicine 2011, 6:4.

15. Xavier MS, Miziara CSMG, Miziara ID. Terminalidade da vida: questões éticas e religiosas sobre a ortotanásia Saúde, Ética \& Justiça. 2014;19(1);26-34. 